Article

\title{
Electrospray Quadrupole Travelling Wave Ion Mobility Time-of-Flight Mass Spectrometry for the Detection of Plasma Metabolome Changes Caused by Xanthohumol in Obese Zucker (fa/fa) Rats
}

Samanthi I. Wickramasekara ${ }^{1}$, Fereshteh Zandkarimi ${ }^{1}$, Jeff Morré ${ }^{1}$, Jay Kirkwood ${ }^{2}$, LeeCole Legette $^{2,3}$, Yuan Jiang ${ }^{4}$, Adrian F. Gombart ${ }^{3,5}$, Jan F. Stevens ${ }^{2,3}$ and Claudia S. Maier ${ }^{1, *}$

1 Department of Chemistry, Oregon State University, Corvallis, OR 97331, USA;

E-Mails: samanthi.wickramasekara@oregonstate.edu (S.I.W.); zandkarf@onid.orst.edu (F.Z.); jeff.morre@oregonstate.edu (J.M.)

2 Department of Pharmaceutical Sciences, Oregon State University, Corvallis, OR 97331, USA;

E-Mails: kirkwooj@onid.orst.edu (J.K.); legettel@onid.orst.edu (L.L.);

fred.stevens@oregonstate.edu (J.F.S.)

3 Linus Pauling Institute, Oregon State University, Corvallis, OR 97331, USA;

E-Mail: adrian.gombart@oregonstate.edu

4 Department of Statistics, Oregon State University, Corvallis, OR 97331, USA;

E-Mail: yuan.jiang@stat.oregonstate.edu

5 Department of Biochemistry and Biophysics, Oregon State University, Corvallis, OR 97331, USA

* Author to whom correspondence should be addressed;

E-Mail: claudia.maier@oregonstate.edu; Tel.: +1-541-737-9533; Fax:+1-541-737-2062.

Received: 3 June 2013; in revised form: 1 August 2013 / Accepted: 7 August 2013 /

Published: 13 August 2013

Abstract: This study reports on the use of traveling wave ion mobility quadrupole time-of-flight (ToF) mass spectrometry for plasma metabolomics. Plasma metabolite profiles of obese Zucker fa/fa rats were obtained after the administration of different oral doses of Xanthohumol; a hop-derived dietary supplement. Liquid chromatography coupled data independent tandem mass spectrometry $\left(\mathrm{LC}-\mathrm{MS}^{\mathrm{E}}\right)$ and $\mathrm{LC}$-ion mobility spectrometry (IMS)-MS ${ }^{\mathrm{E}}$ acquisitions were conducted in both positive and negative modes using a Synapt G2 High Definition Mass Spectrometry (HDMS) instrument. This method provides identification of metabolite classes in rat plasma using parallel alternating low energy and 
high energy collision spectral acquisition modes. Data sets were analyzed using pattern recognition methods. Statistically significant $(p<0.05$ and fold change $(\mathrm{FC})$ threshold $>1.5)$ features were selected to identify the up-/down-regulated metabolite classes. Ion mobility data visualized using drift scope software provided a graphical read-out of differences in metabolite classes.

Keywords: obesity; xanthohumol; travelling wave ion mobility; mass spectrometry; lipidomics

\section{Introduction}

Metabolomics, the analysis of endogenous metabolites $(<1,500$ Dalton) in complex mixtures, is heavily based on the combination of separation techniques and mass spectrometry. The predominant analytical methods are hyphenated approaches combining gas chromatography with mass spectrometry (GC-MS) [1,2], liquid chromatography and mass spectrometry (LC-MS) [3,4] and capillary electrophoresis and mass spectrometry (CE-MS) [5-7]. As an analytical science, advances in metabolomics are predominately achieved by technology advancements [8,9]. During the last decade, mass analyzers have seen significant improvements in resolving power, mass accuracy and sensitivity. The combination of soft ionization techniques, such as atmospheric pressure chemical ionization (APCI) and electrospray ionization (ESI), and hyphenated LC-MS/MS techniques are the current method of choice for the analysis of polar metabolites [10,11].

The precise and accurate quantification of metabolites is the key objective of targeted metabolomics. Targeted metabolite profiling analyses are often conducted using triple quadrupole instruments operated in the selected reaction monitoring mode. Q-trap-type instruments allow data-dependent acquisition modes that combine selected reaction monitoring and product ion scanning modes, providing good quantification of analytes and increased confidence in metabolite identification $[4,12,13]$. In contrast, the main objective of untargeted metabolomics is the detection and identification of as many metabolites as possible. Accurate mass measurement of the metabolites is of paramount importance for the success of untargeted metabolome analyses, because the mass-to-charge ratio $(\mathrm{m} / \mathrm{z})$ value of the metabolite ion is used initially for searching metabolite databases. Current mass analyzers that enable accurate mass measurements with high resolution are modern time-of-flight (ToF) instruments, Fourier transform (FT) orbitraps and ion cyclotron resonance (ICR) analyzers. However, if metabolite identification is the goal of the analysis, the mass information needs to be complemented with structural data that can be obtained by performing collision-induced dissociation (CID). The most common acquisition mode in untargeted metabolomic experiments is the acquisition of full scan data and product ion data using data-dependent acquisition (DDA) modes [14,15]. DDA acquisitions are technically realized in hybrid tandem mass spectrometry instruments by combining multiple mass analyzer, such as the Q-ToF-type instruments, quadrupole orbitraps (Q Exactive), and combinations of linear trap quadrupole (LTQ) instruments with high resolution mass analyzer, e.g. LTQ-orbitraps and the LTQ-FT instruments. A possible drawback of DDAs is that a substantial proportion of the acquisition cycle is spent acquiring product ion spectra; therefore, the accuracy with which the intensities of the precursor ions are measured in the first MS stage (MS1) are compromised [16]. More 
recently, data-independent acquisition (DIA) methods have become available that do not select specific precursor ions for gas phase fragmentation and acquire ion signals for precursor and fragment ion seemingly uninterrupted. It is for this reason that DDA methods deliver more accurate quantitative precursor ion signals suited for quantitative studies [17,18]. Independent of the mode of acquisition, untargeted metabolomic experiments greatly benefit from the availability of high resolution accurate mass measurements of precursor and product ions, enabling the assignments of metabolites with greater confidence.

Recent developments in quadrupole time-of-flight (q-ToF) hybrid instruments led to improvements in sensitivity, duty cycle and dynamic range. In addition, the introduction of collision energy profiles for the non-selective MS/MS acquisition mode, so-called $\mathrm{MS}^{\mathrm{E}}$, provides an untargeted and unbiased methodology for metabolite fragmentation $[17,19,20]$. The combination of $\mathrm{MS}^{\mathrm{E}}$ acquisitions with the separation power of Ultra Performance Liquid Chromatography (UPLC) enables conducting many precursor and neutral loss acquisitions over a single experimental run and can overcome duty cycle issues associated with similar types of experiments involving other scanning-type instruments $[21,22]$. Furthermore, $\mathrm{MS}^{\mathrm{E}}$ supports the analysis of any compound of interest irrespective of its signal intensity and has been applied for many proteomic and metabolomic studies [17,23-25]. Additionally, $\mathrm{MS}^{\mathrm{E}}$ in conjunction with a high-end ToF analyzer provides exact mass information for precursor and fragment ions, a prerequisite for determining elemental compositions and minimizing false positive identifications.

The combination of ion mobility spectrometry (IMS) with hybrid mass spectrometry (MS) instruments offers an additional dimension of separation for complex mixtures. In ion mobility spectrometry, ions are separated as they drift through gas $\left(\mathrm{He}, \mathrm{Ar}, \mathrm{CO}_{2}\right)$ under the influence of an electric field. The rates of drift depend on the size, charge and the collisional cross section of the respective ions. Adding the drift time information may add an additional level of specificity to the mass-to-charge $(\mathrm{m} / \mathrm{z})$ analysis. The integration of ion mobility spectrometry with mass spectrometry (IMS-MS) provides a rapid method for the analysis of isobaric molecules, such as small molecule regioisomers and polypeptides adopting different conformer structures [26-28]. In the current report, a commercial ion mobility mass spectrometry instrument that features a Triwave ${ }^{\mathrm{TM}}$ geometry in which the ion mobility cell (IMS T-wave) is placed between two traveling wave ion guides (trap T-wave and transfer T-wave) was used. These devices can be used as either ion guides or collision cells, depending on the collision energy voltages applied. Fragmentation of ions can be induced in both devices, the trap (pre-IMS separation) and/or the transfer (post-IMS separation) [29]. With the trap-only fragmentation, the ions will undergo high energy collisions before they enter into the IMS T-wave region. Then, the fragments will be separated in the second travelling wave device based on their collisional cross sections and be guided to the ToF detector with minimum or no collision at the transfer T-wave cell. With the transfer-only fragmentation, the ions pass through the first travelling wave cell with no collision energy applied, and the parent ions will separate by the IMS T-wave cell, followed by fragmentation in the transfer T-wave region. When the ions fragment in this region, they retain the original drift time of the parent ion., The combined use of both retention time- and drift time-alignment of parent and fragment ions enables the identification of metabolites with high confidence even in complex matrices. Another way to get more structural information on the ions is to use time-aligned parallel (TAP) fragmentation, in which both trap T-wave and transfer T-wave cells are kept at high voltage. In the TAP mode, the ion of interest will be selected in the quadrupole region and will 
undergo first generation fragmentation in the trap cell, and the resulting fragment ions will separate in the ion mobility cell. The mobility-separated fragment ions will undergo further fragmentation, resulting in second generation fragment ions, which are time-aligned with the first generation fragment ions [26]. In this study, we used ion mobility separation in combination with $\mathrm{MS}^{\mathrm{E}}$ acquisition for the identification of plasma metabolites from obese Zucker rats. In this method, precursor ions are separated by ion mobility and, then, subjected to $\mathrm{MS}^{\mathrm{E}}$ acquisition, which applies parallel low and high energy cycles for achieving exact mass analyses and fragment ion generation. In this mode, the fragment ions are aligned with their precursors with respect to their drift times.

Xanthohumol $(\mathrm{XN})$, a prenyl flavonoid extracted from the hop plant, has gained much attention, due to its anti-inflammatory, antimicrobial, antioxidant and anticancer properties [30,31]. Work by Legette et al. indicated that the XN lowers body weight and improved glucose homeostasis in obese Zucker (fa/fa) rats [32]. As part of a larger ongoing effort to investigate the possible health promoting effects of $\mathrm{XN}$ in obesity, the objective of this study was to apply electrospray travelling wave ion mobility mass spectrometry (ESI-T-wave-IMS-MS) to investigate the plasma metabolome of obese Zucker (fa/fa) rats treated with different oral doses of xanthohumol.

\section{Results and Discussion}

\subsection{HPLC-TOF-MS Analysis}

Identification of Up-/Down -Regulated Metabolites

Plasma samples from both untreated or treated (low, medium or high dose xanthohumol) male and female Zucker rats were analyzed by UPLC-MS ${ }^{\mathrm{E}}$ and UPLC-IMS-MS ${ }^{\mathrm{E}}$ in positive and negative mode. Initially, we used unsupervised principal component analysis (PCA) to evaluate the data sets and to obtain a first indication of general separation trends. This indicated that groups were separated by gender (Supplemental Materials, Figure S1); therefore, we evaluated female and male treatment groups separately. The PCA scores plot generated from the female rat data sets of all four groups (control and three xanthohumol treatment groups) indicated that the xanthohumol treatment groups showed little separation tendency, whereas the data sets of the male rats showed clear separation between control and the different dose groups (Supplemental Materials, Figure S2). This observation is consistent with previous findings by Legette et al. that showed XN treatment caused loss of bodyweight in male, but not in female, obese Zucker rats [32].

We, therefore, concentrated our metabolomic analyses on the data sets obtained from the male rat plasma samples of the control group $(n=6)$ and the group treated with the high dose of xanthohumol $(n=6)$. Pre-processing of the data using XCMS, an open-source metabolomic data processing software (http://metlin.scripps.edu/xcms/), resulted in a three-dimensional matrix, mass-to-charge ratio $(\mathrm{m} / \mathrm{z}$ ), retention time (RT) and intensity values, consisting of 4,331 and 4,019 features in the positive and negative mode, respectively. These features were used for both univariate and multivariate data analysis. The scores plot showed a separation tendency between the control and the treatment group. Consequently, a Partial Least Square-Discriminant Analysis (PLS-DA) was performed to identify discriminating features between the groups. As shown in Figure 1, top left, the control group clustered well and is separated from the treatment (high dose) group; however, treated samples were spread out. Student $t$ - 
tests were performed for statistical comparisons between the control and treated group, and $p$-values of 0.05 or less were considered significant. Further visualization of data was carried out using a heat map with two way-hierarchical clustering using the Ward algorithm and Euclidian distance on the top 50 discriminating features between the two study groups. This hierarchical clustering resulted in better separation of the control group from the treated group by reducing the variation in the treated group (Figure 1A, B). Finally, the candidate markers were selected by examining the volcano plot and considering a fold change threshold of 1.5 and a $p$-value less than 0.05 (Supplemental Materials, Figure S3). In addition, $\mathrm{XN}$ and $\mathrm{XN}$-related metabolites [31,33] were excluded from the candidate marker list before performing the database searches. A total of 121 features were selected for accurate mass-based annotation using the Metlin, Lipidmaps, Madison Metabolomics Consortium Database (MMCD) and Human Metabolome Database (HMDB) databases. Differential metabolites are listed in the Supplemental Materials, Table 1.

Figure 1. Partial Least Square-Discriminant Analysis (PLS-DA) plot (A, B) and unsupervised hierarchical clustering plot $(\mathbf{C}, \mathbf{D})$ for control and high dose groups (male animals). (A, C) positive ionization mode; (B, D) negative ionization mode; a heat map was constructed using the top 50 important metabolites created from MetaboAnalyst software (2.0). The metabolites and samples were hierarchically clustered by the Ward algorithm using Euclidian distance. Each column represents a unique feature with a characteristic mass-to-charge ratio and retention time value. As shown in the heat map alignment, the two groups were clustered by an unsupervised algorithm, which confirms the presence of discriminating features between the high dose and control groups.

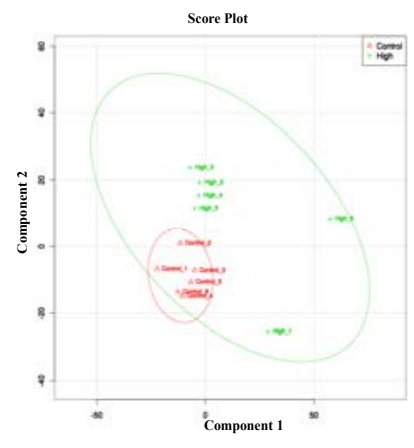

A

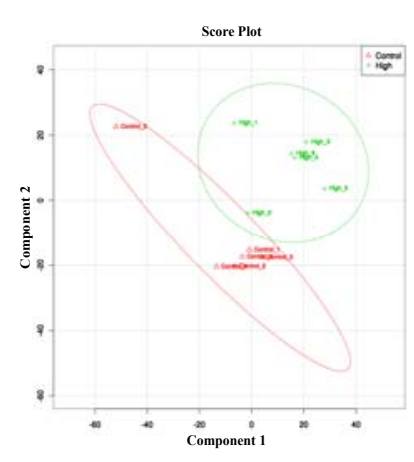

C

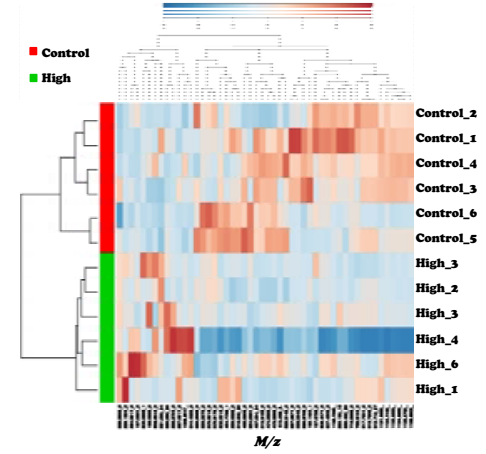

C

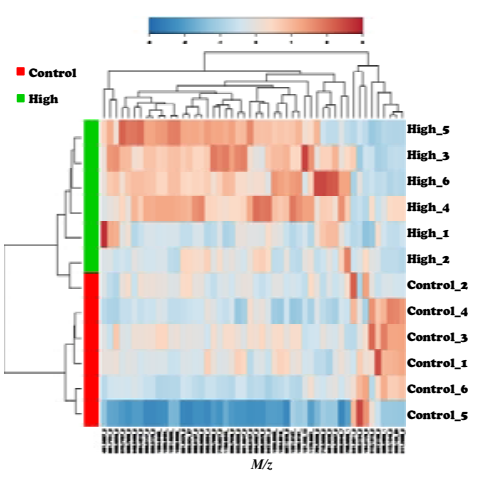

D 
In the current study we utilized the $\mathrm{MS}^{\mathrm{E}}$ mode, which acquires low energy full scan spectra and high energy fragmentation spectra in a single run. $\mathrm{MS}^{\mathrm{E}}$ data provides access to exact mass information for precursor, the fragment ion and neutral loss data to search for diagnostic ions or common neutral losses. In the current study, we focused on the impact of xanthohumol treatment on lipid species. Utilization of $\mathrm{MS}^{\mathrm{E}}$ allowed us to extract mass spectral data of distinct lipid classes based on class-specific fragment ions. For example, Figure $2 \mathrm{~B}$ shows the extracted ion chromatogram (XIC) for an $\mathrm{m} / \mathrm{z}$ of 184.07, characteristic for the phosphocholine head group, which enabled the extraction of elution profiles for the following lipid classes: lysophosphatidyl choline (Lyso-PC), phosphatidyl cholines (PC) and sphingomyelin (SM) lipids. Accurate mass measurements and high mass spectral resolution in both the low and the high energy mode allowed for higher confidence in metabolite assignments.

Figure 2. (A) Full scan mass spectrometry (MS) chromatograms obtained in $\mathrm{MS}^{\mathrm{E}}$ mode using low (top) and high (bottom) energy regimes. Extracted ion chromatogram (XIC) obtained with an $\mathrm{m} / \mathrm{z}$ of 184.0740 (phosphocholine head group) enables localization of the elution window for lysophosphatidyl choline (Lyso-PC), phosphatidyl cholines (PC) and sphingomyelin (SM) lipid classes. (B) Extracted mass spectra of low- (top) and high- (bottom) collision energy for [Lyso-PC $(16: 0)+\mathrm{H}]^{+}-\left(\mathrm{C}_{24} \mathrm{H}_{51} \mathrm{NO}_{7} \mathrm{P}\right)$. The low-energy spectrum contains the precursor ion at $m / z 496.3417\left([\mathrm{M}+\mathrm{H}]^{+}\right)$, whereas the high-energy spectrum is dominated by the fragment ion for the phosphocholine head group $(m / z$ 184.0740).

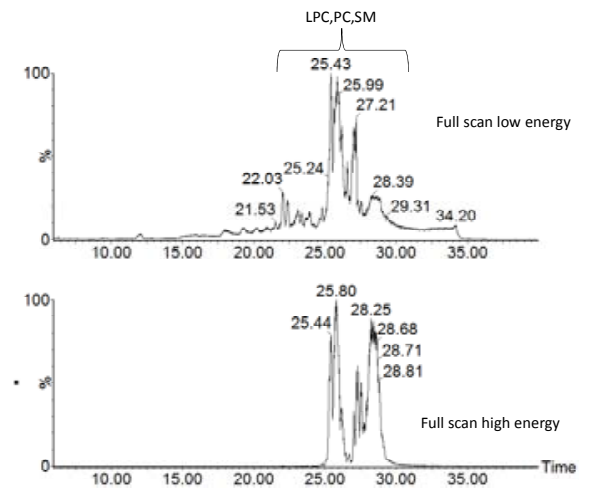

A
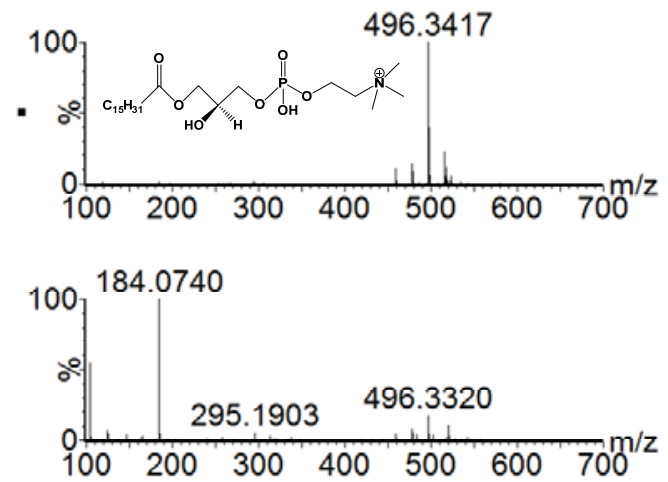

B

Lipid identifications were carried out by the Metabosearch software with four embedded metabolite databases. There were many $\mathrm{m} / \mathrm{z}$ values that did not match any of the database entries. This is a common phenomenon associated with untargeted metabolomic data and is at least partly attributable to possible adduct formation $\left(\mathrm{Na}^{+}, \mathrm{NH}_{4}^{+}\right.$) and/or in-source fragmentation (loss of $\mathrm{H}_{2} \mathrm{O}$, loss of $\mathrm{CO}_{2}$ ). Lipids that showed significant fold changes (Student $t$-test, $p<0.05$ ) are compiled in Table 1. The observed changes of different lipid classes suggest that xanthohumol affects lipid metabolism.

Based on the fold-change values obtained with the treated vs. control group, the level of plasma fatty acyl groups increased in the treated group. In contrast, plasma phosphatidic acid (PA) levels were lower in the treated animals compared to the controls. PAs are important intermediates in the biosynthesis of triacylglycerols and phospholipids and play an important role as signaling molecules [34]. 
Noteworthy, most of the phosphatidic acids were detected as Lyso-PA species. It was reported that the level and the composition of Lyso-PA changes rapidly due to external stimulants and inflammatory diseases. Lyso-PAs have been linked to metabolic defects associated with obesity [32,35]. Obesity is a chronic inflammatory disease. Under conditions of inflammation, phospholipases are activated and hydrolyze the sn-1 (PLA 1$)$ or sn-2 (PLA $)$ acyl bond of phospholipids [36,37]. The ratio of PC/Lyso-PC has been reported as an indicator for inflammation [38]. We examined the sum of the normalized response of the PC/Lyso-PC ratio for the control and high dose group, and about a $10 \%-20 \%$ variation for the PC/Lyso-PC ratio was observed with the high dose treatment. For example, the fold change (treated/control) for the PC/Lyso-PC ratio between PC (18:0/18:3) and Lyso-PC (18:0) was calculated to be 1.2 , which suggests that high dose treatment with xanthohumol reduced inflammation.

Table 1. Differential lipids in control vs. treated groups. Metabolites with identification probability $<0.05$ and a fold change $(\mathrm{FC})$ threshold $>1.5$, with a mass error $<5 \mathrm{ppm}$, were selected as significant features in both positive and negative ionization modes. Comparison of the distributions of differential lipids within the control and high treatment groups are provided as box-and-whisker plots in Supplementary Figure 4. Putative compound identifications were based on the accurate mass measurements and confirmed by high energy fragmentations.

\begin{tabular}{|c|c|c|c|c|c|}
\hline Putative metabolites & $\begin{array}{l}\text { Up-/down- } \\
\text { regulation } \\
(\mathrm{T} / \mathrm{C})\end{array}$ & $m / z$ & $\begin{array}{l}\text { Molecular } \\
\text { formula }\end{array}$ & $\begin{array}{l}\text { Mass error } \\
\quad(\mathbf{p p m})\end{array}$ & $\begin{array}{l}\text { Detected } \\
\text { fragments }\end{array}$ \\
\hline Hexadecanedioic acid & $\uparrow$ & 285.206 & $\mathrm{C}_{16} \mathrm{H}_{30} \mathrm{O}_{4}$ & -2.45 & {$\left[\mathrm{M}-\mathrm{H}-\mathrm{H}_{2} \mathrm{O}\right]^{-}$} \\
\hline Lauroyl-EA & $\downarrow$ & 244.228 & $\mathrm{C}_{14} \mathrm{H}_{29} \mathrm{NO}_{2}$ & 2.87 & {$\left[\mathrm{M}+\mathrm{H}-\mathrm{H}_{2} \mathrm{O}\right]^{+}$} \\
\hline Octadecanedioic acid & $\uparrow$ & 313.237 & $\mathrm{C}_{18} \mathrm{H}_{34} \mathrm{O}_{4}$ & -3.51 & {$\left[\mathrm{M}-\mathrm{H}-\mathrm{H}_{2} \mathrm{O}\right]^{-}$} \\
\hline $\operatorname{PA}(18: 1 / 0: 0)$ & $\downarrow$ & 435.254 & $\mathrm{C}_{21} \mathrm{H}_{41} \mathrm{O}_{7} \mathrm{P}$ & 4.82 & {$[\text { FA } 18: 1-\mathrm{H}]^{-}$} \\
\hline $\operatorname{PA}(18: 2 / 0: 0)$ & $\downarrow$ & 433.236 & $\mathrm{C}_{21} \mathrm{H}_{39} \mathrm{O}_{7} \mathrm{P}$ & -1.15 & {$[\text { FA } 18: 2-\mathrm{H}]^{-}$} \\
\hline $\operatorname{PA}(20: 4 / 0: 0)$ & $\downarrow$ & 457.235 & $\mathrm{C}_{23} \mathrm{H}_{39} \mathrm{O}_{7} \mathrm{P}$ & -3.5 & $153\left[\mathrm{C}_{3} \mathrm{H}_{6} \mathrm{PO}_{5}\right]^{-}$ \\
\hline $\mathrm{PA}(\mathrm{O}-16: 0 / 18: 2)$ & $\downarrow$ & 657.485 & $\mathrm{C}_{37} \mathrm{H}_{71} \mathrm{O}_{7} \mathrm{P}$ & -1.67 & {$[\text { FA } 18: 2-\mathrm{H}]^{-}$} \\
\hline $\operatorname{PG}(18: 1 / 0: 0)$ & $\downarrow$ & 509.287 & $\mathrm{C}_{24} \mathrm{H}_{47} \mathrm{O}_{9} \mathrm{P}$ & -2.55 & $\begin{array}{l}\left(153\left[\mathrm{C}_{3} \mathrm{H}_{6} \mathrm{PO}_{5}\right]^{-}\right), \\
{[\mathrm{FA} 18: 1-\mathrm{H}]^{-}}\end{array}$ \\
\hline PI(18:3/0:0) & $\uparrow$ & 593.273 & $\mathrm{C}_{27} \mathrm{H}_{47} \mathrm{O}_{12} \mathrm{P}$ & 0.34 & $153\left[\mathrm{C}_{3} \mathrm{H}_{6} \mathrm{PO}_{5}\right]^{-}$ \\
\hline $\mathrm{PI}(\mathrm{P}-18: 0 / 22: 4)$ & $\uparrow$ & 897.583 & $\mathrm{C}_{49} \mathrm{H}_{87} \mathrm{O}_{12} \mathrm{P}$ & -3.57 & $153\left[\mathrm{C}_{3} \mathrm{H}_{6} \mathrm{PO}_{5}\right]^{-}$ \\
\hline $\operatorname{PS}(18: 4 / 22: 6)$ & $\downarrow$ & 826.464 & $\mathrm{C}_{46} \mathrm{H}_{70} \mathrm{NO}_{10} \mathrm{P}$ & -3.62 & $\begin{array}{l}\left(153\left[\mathrm{C}_{3} \mathrm{H}_{6} \mathrm{PO}_{5}\right]^{-}\right), \\
{[\text {FA 22:6-H] }}\end{array}$ \\
\hline $\operatorname{PS}(20: 1 / 22: 6)$ & $\downarrow$ & 862.559 & $\mathrm{C}_{48} \mathrm{H}_{80} \mathrm{NO}_{10} \mathrm{P}$ & -0.35 & {$[\mathrm{M}+\mathrm{H}-185]^{+}$} \\
\hline $\begin{array}{l}\text { Sphingosine-1- } \\
\text { phosphate }\end{array}$ & $\downarrow$ & 378.24 & $\mathrm{C}_{18} \mathrm{H}_{38} \mathrm{NO}_{5} \mathrm{P}$ & -4.49 & $\begin{array}{l}\left(79\left[\mathrm{PO}_{3}\right]^{-}\right),(97 \\
\left.\left[\mathrm{H}_{2} \mathrm{PO}_{4}\right]^{-}\right)\end{array}$ \\
\hline
\end{tabular}

$\mathrm{T} / \mathrm{C}$ - treated $v s$. control; $m / z$ — mass to charge ratio; PA—acylglycerophosphate; $\mathrm{PG}$-phospho-glycerol; PI—phospho-inositol; PS—phospho-serine; EA—ethanol amine; FA—fatty acid.

Other lipid classes that appeared to be significantly dysregulated in the treated groups are glycerophospho-glycerols (PG), phosphatidyl serines (PS) and glycerophospho-inositols (PI). Taken together, the metabolome changes suggest that xanthohumol in the obese Zucker (fa/fa) rat alters the levels of circulating phospholipids. Up-/down-regulation of these lipid classes in the xanthohumol-treated group is consistent with the findings in a previous study that reported that xanthohumol alters cholesterol 
and bile acid metabolism, as well as lowers the plasma triglyceride levels of obese rats [39]. Dicarboxylic fatty acids are the products of fatty acid metabolism and can be found in high abundance when mitochondrial beta oxidation is dysfunctional [40]. We found significantly decreased levels of dicarboxylic acids in the plasma samples from xanthohumol-treated animals, which may support the notion that xanthohumol treatment enhances the mitochondrial beta oxidation process. These findings agree with the recently published rat plasma and cell culture studies conducted by Kirkwood et al. with similar XN treatments [41].

\subsection{Traveling Wave Ion Mobility Mass Spectrometry (LC-IMS-MS E Analysis)}

During the last few years, there has been a steady increase in the use of ion mobility mass spectrometry (IMS-MS) for the rapid separation of metabolites in complex mixtures [42]. Technical advancement in instrument design and modern electronics has enabled continuous improvements in the resolution of the ion mobility device. The combination of ion mobility separators and high resolution mass analyzers is poised to impact and advance the field of metabolomics [12]. The inclusion of ion mobility separations to complex mixture analyses is twofold: first, ion mobility separation expands the separation space by adding an orthogonal dimension of separation to chromatographic separations; second, ion mobility spectrometry enables the extraction of collisional cross sections and, hence, provides an additional level of specificity to structure elucidations. The number of applications of IMS-MS for the advanced structure analyses of metabolites of small molecule drugs is growing and includes the elucidation of the hydroxylation and glucuronidation patterns of small molecule drugs [43], applications to untargeted metabolome analyses [44], lipid analyses [45,46] and carbohydrates [47-49]. In this report, we applied travelling wave ion mobility in combination with $\mathrm{MS}^{\mathrm{E}}$ acquisition (IMS-MS ${ }^{\mathrm{E}}$ ) to reduce the congestion of ion signals of lipids that were insufficiently separated under the applied LC conditions.

Figure 3 shows an example of a 2D image plotted with drift time vs. retention time, for a plasma sample from the xanthohumol (high dose)-treated group. Ion mobility separation facilitated the detection of low abundant lipid ions by separating the lipid ions from matrix ions and other spectral interferences. As described earlier, most of the lipid classes with a polar phosphocholine head group co-eluted, because of the relatively short LC gradient used in this study. The additional separation space provided by ion mobility enabled the separation of lipid classes that co-eluted at the chromatographic scale, leading to mass spectral overlap and data crowding. As shown in Figure 3, post-acquisition spectral extraction of the two regions marked in the $2 \mathrm{D}$ image enabled the interrogation of the distinct lipid classes with minimal spectral interference. Based on the accurate mass measurement, the ion cluster with a drift time distribution centered on seven milliseconds belongs to Lyso-PC lipids, whereas the SM lipids show a drift time distribution around 4.75 milliseconds. PC and SM share the same characteristic fragment ion with the loss of the polar phosphocholine head group ( $m / z$ 184.0739). The main difference between these two lipid classes is the linkage with the phosphatidyl head group. In SM lipids, the head group is linked via an $\mathrm{N}$-acyl fatty acid group with a long chain hydrocarbon [18]. In a regular $\mathrm{MS}^{\mathrm{E}}$ experiment, some of the information of these co-eluting compounds can be lost due to the differences in ion abundance. Separating these compounds in an additional dimension enabled the identification of more low abundance ions and provided relatively "cleaner" spectra for both MS and MS/MS analyses. Figure 4 illustrates the high energy fragmentation of selected Lyso-PCs isolated from drift time region 2 (see Figure 3). Collision energy ramping from 
15-45 eV was applied to fragment "all" ions entering into the transfer region, and the resulting spectra showed the characteristic fragmentation pattern with the loss of the phosphatidyl choline head group $(\mathrm{m} / \mathrm{z}$ 184.0740) as the dominating ion in all the samples. With the application of high energy, the phosphatidyl head group fragmented further to give another characteristic ion with $\mathrm{m} / z 104.1041$ $\left(\left[\mathrm{C}_{5} \mathrm{H}_{14} \mathrm{NO}\right]^{+}\right)$. Fragmenting after IMS generates product ions that are drift time- and retention time-aligned, with their precursors providing more confident metabolite identifications with low or only minimal matrix interferences.

Figure 3. An example of a $2 \mathrm{D}$ image (drift time $v s$. retention time) showing the ion mobility separation of different compound classes in rat plasma samples. The encircled regions mark the compound classes that eluted within a similar retention time window (26-28 min). Drift time-extracted spectra (bottom) show that these two clusters belong to different lipid classes, namely Lyso-PC and SM lipids (sphingosine phosphocholines) that have drift time distributions centered around $7.02 \mathrm{~ms}$ and $4.75 \mathrm{~ms}$, respectively.
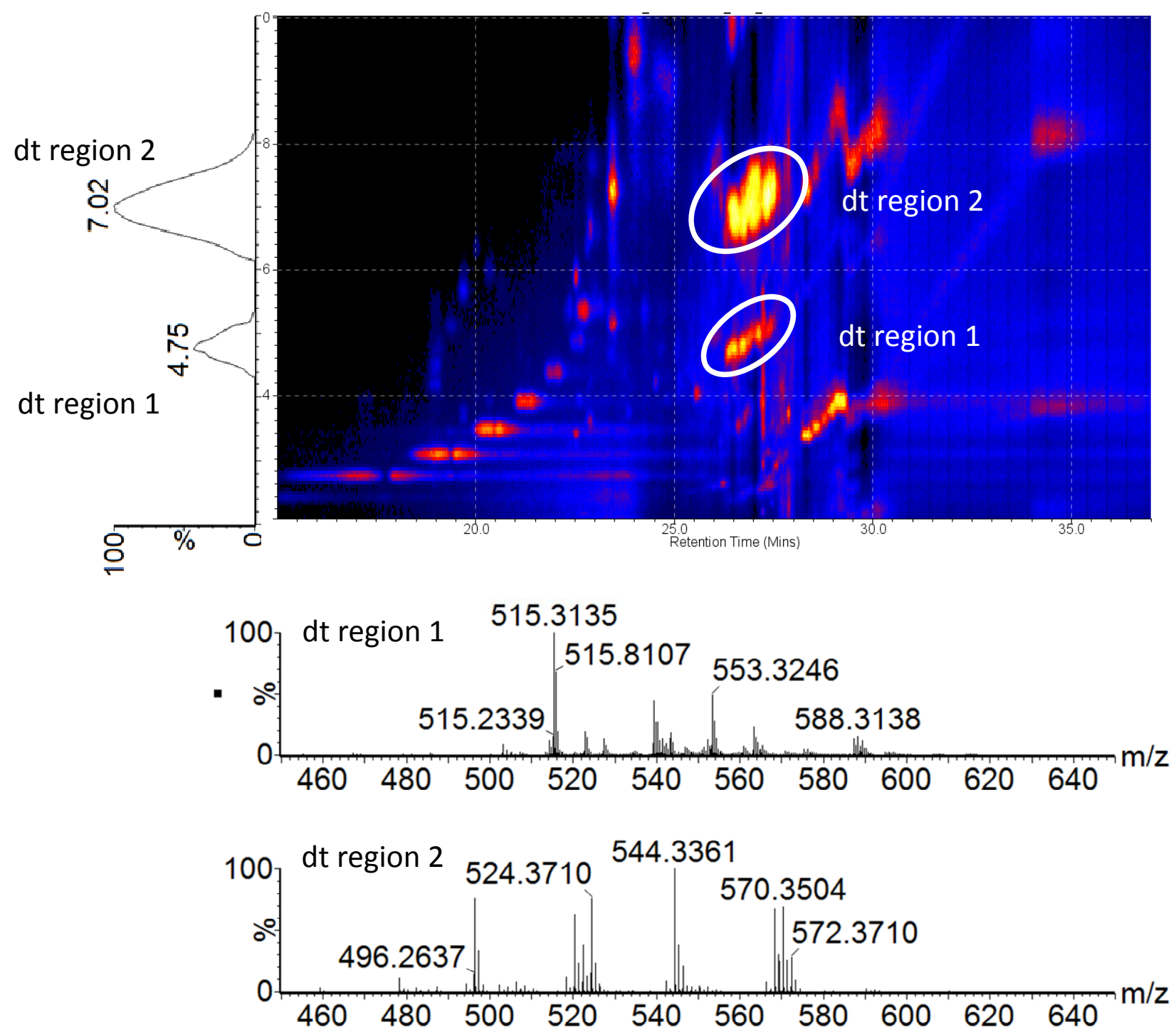
Figure 4. MS/MS spectra extracted from the high energy ion mobility spectrometry (IMS) function corresponding to drift time region $2(\sim 7.0 \mathrm{~ms})$, which contains different Lyso-PCs. The general structure and the predicted fragmentation positions are given at the top. Both saturated and unsaturated ions give rise to the same characteristic fragment ion in high energy mode. The top right corner of each spectrum contains the molecular formula for the selected Lyso-PC ion.

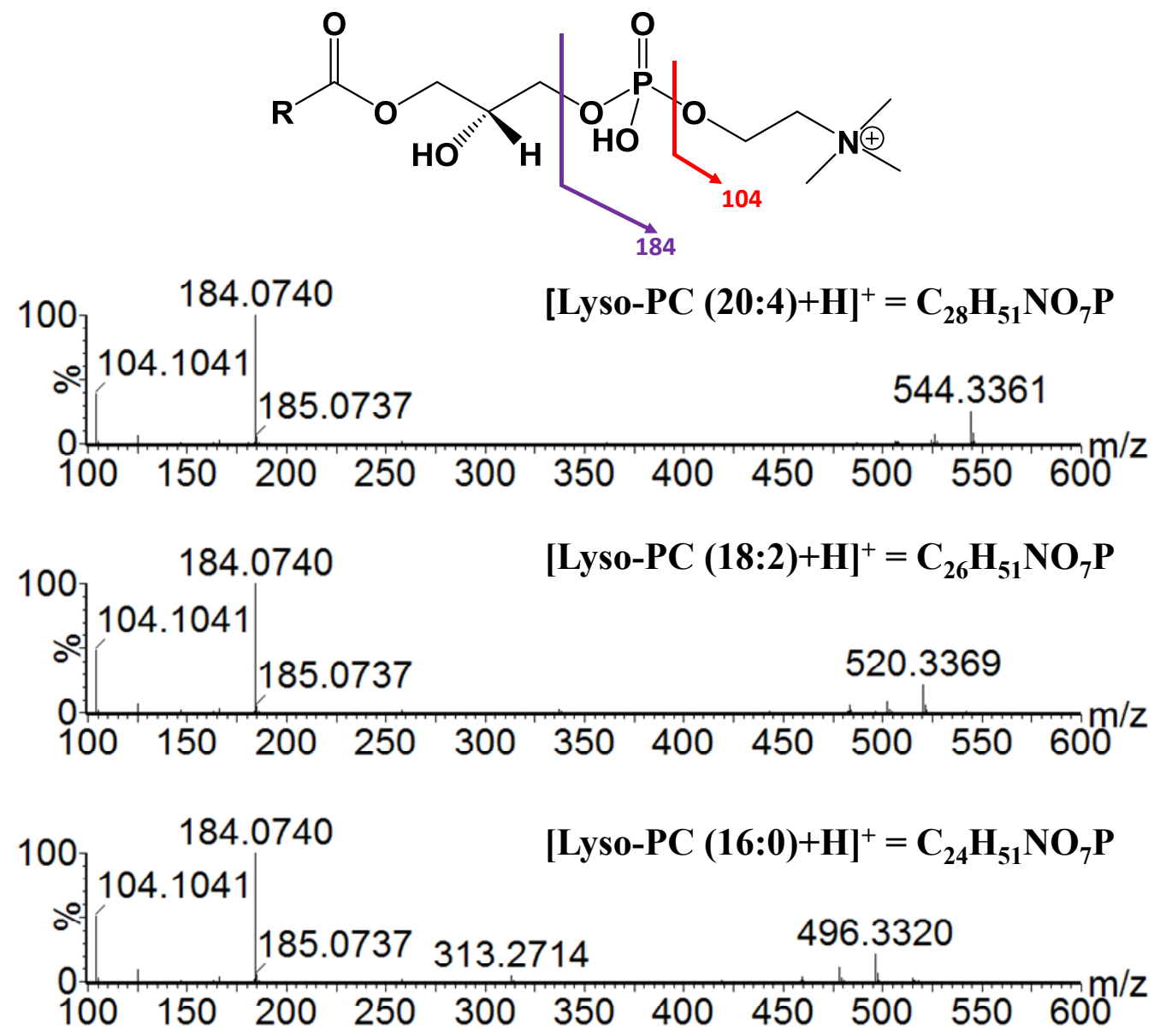

Ion Mobility-Mass Correlation Analyses

After identifying the masses, ion mobility correlations were analyzed for various metabolite classes identified in the Zucker rat (fa/fa) plasma samples. Figure 5 shows the correlation plots with $\mathrm{m} / \mathrm{z}$ on the $\mathrm{x}$-axis and drift time on the $\mathrm{y}$-axis. A total of five compound classes were assigned for significant metabolites detected in the negative and positive ionization modes. These correlation analyses can be used as a predictive tool to determine the compound class for an unknown metabolite. If a compound class database can be created (varying carbon chain lengths, number of double bonds) using a set of standards, these correlation analyses can be used as an additional tool for the verification of a metabolite with regard to using the $\mathrm{m} / \mathrm{z}$ value alone. One of the caveats of this method is the occurrence of multiple charge state molecules. Multiply charged ions overlap with one another in the three-dimensional conformational space and decrease overall peak capacity. This will result in one analyte being represented by several ion signals in the spectrum. Additionally, different adduct formations can lead to false identification of compound classes with the same $m / z$-drift time pairs. 
Figure 5. Ion mobility correlation analyses for the significant features identified in (A) positive and (B) negative ionization modes. Data were acquired in low energy IMS-MS ${ }^{\mathrm{E}}$ mode, and the tentative compound identifications were obtained based on their accurate mass measurements.

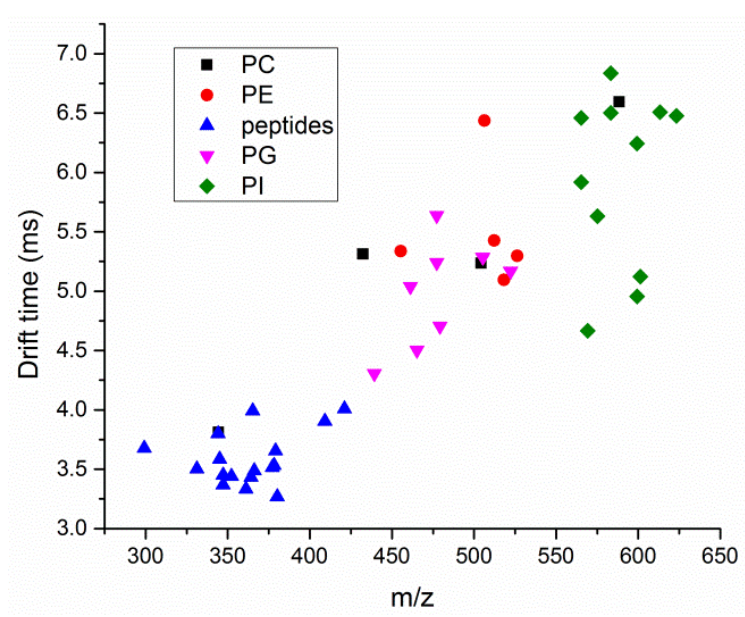

A

PC - phosphatidyl cholines; PG-phospho-glycerol; EA—ethanol amine; FA—fatty acid

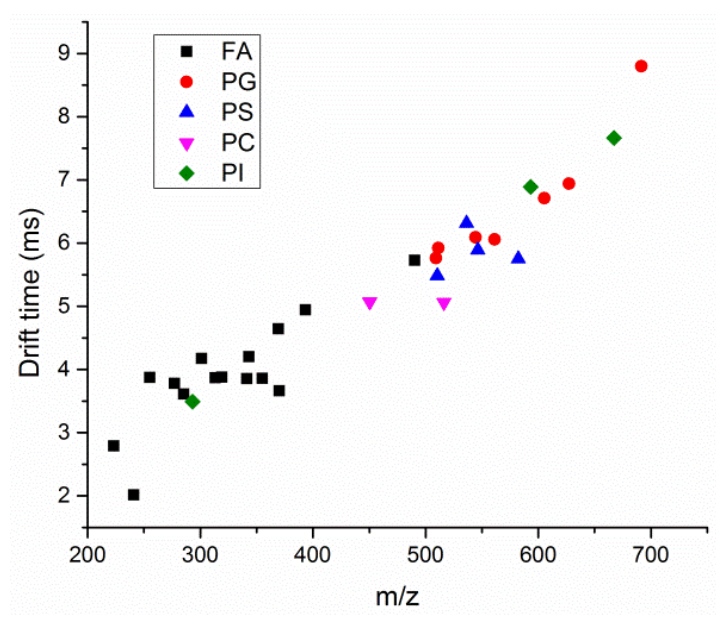

B

PI—phospho-inositol; PS—phospho-serine;

\section{Experimental Section}

\subsection{Sample Preparation}

Samples were prepared according to the method described by Kirkwood et al. [41]. Briefly, four-week old male and female Zucker fa/fa rats were purchased from Harlan (Livermore, CA, USA). All rats were maintained on a high fat $(60 \% \mathrm{kcal})$ AIN-93G diet for 3 weeks to induce severe obesity, followed by a normal AIN- $93 \mathrm{G}(15 \% \mathrm{kcal}$ fat $)$ diet for the last 3 weeks of the study. After a 2-day acclimation period following transportation, 48 animals (24 males and 24 females) were divided into four treatment groups $(\mathrm{n}=6 /$ gender group $)$ : control $(0 \mathrm{mg}$ xanthohumol $/ \mathrm{kg}$ body weight $(\mathrm{BW}))$, low (1.86 mg xanthohumol $/ \mathrm{kg} \mathrm{BW}$ ), medium (5.64 mg xanthohumol $/ \mathrm{kg} \mathrm{BW}$ ) and high (16.9 $\mathrm{mg}$ xanthohumol $/ \mathrm{kg} \mathrm{BW}$ ). Animals were euthanized with an overdose of $\mathrm{CO}_{2}$. Plasma metabolites were extracted using an ice-cold methanol: ethanol mixture (sample/solvent $=1 / 4)$. Sample extracts were kept in a $-80{ }^{\circ} \mathrm{C}$ freezer prior to LC-MS/MS analysis. A quality control (QC) sample was prepared by pooling $10 \mu \mathrm{L}$ from each of the biological samples.

\subsection{LC-MS Analysis}

\subsubsection{LC Setup}

High-performance liquid chromatography was performed on a Shimadzu Nexera system (Shimadzu, Columbia, MD, USA) coupled to a Synapt G2 HDMS mass spectrometer (Waters, Manchester, UK). Chromatographic separations were carried out on an Inertsil phenyl-3 column $(150 \times 4.6 \mathrm{~mm}, 5 \mu \mathrm{m}$, MetaChem Technologies, Torrance, CA, USA) for positive ion and negative ion analysis. Flow rate was set to $0.4 \mathrm{~mL} / \mathrm{min}$, and the mobile phases consisted of water (A) and methanol (B), both with $0.1 \%$ 
formic acid. The elution gradient was as follows: $0 \mathrm{~min}, 5 \% \mathrm{~B} ; 1 \mathrm{~min}, 5 \% \mathrm{~B} ; 11 \mathrm{~min}, 30 \% \mathrm{~B} ; 23 \mathrm{~min}$, $100 \% \mathrm{~B} ; 35 \mathrm{~min}, 100 \% \mathrm{~B} ; 37 \mathrm{~min}, 5 \% \mathrm{~B}$; and $47 \mathrm{~min}, 5 \% \mathrm{~B}$. Column temperature was held at $70{ }^{\circ} \mathrm{C}$, and the injection volume was $10 \mu \mathrm{L}$.

\subsection{2. $\mathrm{MS}^{\mathrm{E}}$}

The inlet (HPLC system) was coupled to a hybrid quadrupole orthogonal time-of-flight mass spectrometer (SYNAPT-G2 HDMS, Waters, MS Technologies, Manchester, UK). Electrospray positive and negative ionization modes were used for the analysis. A capillary voltage and cone voltage of $-3 \mathrm{kV}$ and $-35 \mathrm{~V}$, respectively, were used in both polarities. The desolvation source conditions were as follows: for the desolvation gas, $550 \mathrm{~L} / \mathrm{h}$ was used, and the desolvation temperature was kept at $400{ }^{\circ} \mathrm{C}$. Data acquisition took place over the mass range of 50-1200 $\mathrm{Da}$ in centroid $\mathrm{MS}^{\mathrm{E}}$ mode. The reference internal calibrant (Leucine enkephalin, $\mathrm{m} / \mathrm{z} 556.2771$ for positive ion mode and $\mathrm{m} / \mathrm{z} 554.2615$ for negative ion mode) was introduced into the lock mass sprayer at a constant flow rate of $10 \mu \mathrm{L} / \mathrm{min}$. Lock mass acquisition was controlled automatically by the acquisition software, and a reference scan was conducted every $10 \mathrm{~s}$ lasting $0.3 \mathrm{~s}$. The mass spectrometer was calibrated with sodium formate in both positive and negative ionization modes. During this acquisition method, the first quadrupole, Q1, is operated in a wide band radio frequency (RF) mode only, allowing all ions to enter the T-wave collision cell. Two discrete and independent interleaved acquisition functions are automatically created. The first function, typically set at $5 \mathrm{eV}$, collects low energy or unfragmented data, while the second function collects high energy or fragmented data typically set by using a collision energy ramp from 15-45 eV. In both instances, Argon gas was used for collision-induced dissociation.

\subsubsection{IMS-MS ${ }^{\mathrm{E}}$}

Similar ionization parameters were used to acquire continuum data in IMS-MS ${ }^{\mathrm{E}}$ mode. The trap, IMS and transfer traveling wave devices were operated with traveling wave amplitudes and velocities of $8.0 \mathrm{~V}$ and $508 \mathrm{~m} / \mathrm{s}, 40.0 \mathrm{~V}$ and $1,000 \mathrm{~m} / \mathrm{s}$ and $3.0 \mathrm{~V}$ and $450 \mathrm{~m} / \mathrm{s}$, respectively. The trap/transfer cells were operated with argon at a pressure of $2.5 \times 10^{-2}$ mbar. Nitrogen as the drift gas was introduced into the IMS cell to maintain a pressure of $3.1 \mathrm{mbar}$. The ToF analyzer was operated in the $\mathrm{V}$-mode with an average mass resolution of $\mathrm{m} / \Delta \mathrm{m} 20,000$ (full-width at half-maximum definition). The ToF pusher and trap-gate frequencies were $18.5 \mathrm{kHz}$ and $92.5 \mathrm{~Hz}$, respectively. Data acquisition and processing were performed using MassLynx (V4.1, SCN714), Driftscope (V2.2) and $\mathrm{MS}^{\mathrm{E}}$ viewer (V1.2).

\subsection{LC-MS Data Preprocessing and Statistical Analysis}

$\mathrm{MS}^{\mathrm{E}}$ data were converted into NetCDF format (Network Common Data Form) using MassLynx software (Waters Corp, Milford, MA). Converted data sets were analyzed by the XCMS program using the $\mathrm{R}$ package (2.15.1) for initial data processing, including peak grouping, retention time alignment and filling in missing features [50]. The resulting data matrices were then imported into MetaboAnalyst software (2.0) for statistical analysis [51,52]. Xanthohumol and its related metabolites were excluded from the sample list prior to statistical validation [31,33]. Features in a data matrix were 
filtered based on relative standard deviation (RSD) of whole intensity values, and log transformation and auto-scaling were incorporated to ensure equal contributions from each variable to the models. Partial Least Square Discriminant Analysis (PLS-DA) was performed to identify discriminating features between control treatment groups. Data visualization using unsupervised, two-way hierarchical clustering was performed using the Ward algorithm using the Euclidian distance on the normalized metabolites. A Student $t$-test was performed for statistical comparison between control and high dose rat samples, and a $p$-value of 0.05 or less was considered significant. Significant features were selected based on the $p$-value $(p<0.05)$ and fold change threshold $(\mathrm{FC}>1.5)$. MetaboSearch was used to perform $\mathrm{m} / \mathrm{z}$-based searches simultaneously against the four major metabolite databases: Human Metabolome Database (HMDB), Madison Metabolomics Consortium Database (MMCD), Metlin and LipidMaps [53]. Finally, the compound assignments were confirmed by matching the high energy $\mathrm{MS}^{\mathrm{E}}$ data with the reported and predicted fragmentation patterns for selected metabolites.

\section{Conclusions}

Application of $\mathrm{MS}^{\mathrm{E}}$ and IMS-MS ${ }^{\mathrm{E}}$ for untargeted metabolomics has been demonstrated for plasma samples obtained from obese Zucker (fa/fa) rats that were treated with different oral doses of xanthohumol. Data-independent $\mathrm{MS}^{\mathrm{E}}$ allowed the acquisition of both low energy and high energy spectra in the same analysis. Addition of ion mobility as an additional separation dimension enabled the detection and identification of low abundant sphingolipids in a congested mass spectral region. Based on the dose response studies, the differential metabolites identified confirmed the anti-inflammatory properties of xanthohumol and further suggest that xanthohumol affects lipid metabolism in obese Zucker (fa/fa) rats.

\section{Acknowledgments}

This work was supported by National Institutes of Health grants R21AT005294 (JFS), R01AI65604 (AFG) and S10 RR025628 (CSM). The authors wish to acknowledge OSU's Mass Spectrometry Facility and core, which is supported in part by a grant from the National Institute of Environmental Health Sciences (P30 ES000210).

The content is solely the responsibility of the authors and does not necessarily represent the official views of NIEHS or NIH.

\section{Conflict of Interest}

The authors declare no conflict of interest.

\section{References}

1. Escobar-Morreale, H.F.; Samino, S.; Insenser, M.; Vinaixa, M.; Luque-Ramirez, M.; Lasuncion, M.A.; Correig, X. Metabolic heterogeneity in polycystic ovary syndrome is determined by obesity: Plasma metabolomic approach using GC-MS. Clin. Chem. 2012, 58, 999-1009. 
2. Lin, S.; Liu, N.; Yang, Z.; Song, W.; Wang, P.; Chen, H.; Lucio, M.; Schmitt-Kopplin, P.; Chen, G.; Cai, Z. GC/MS-based metabolomics reveals fatty acid biosynthesis and cholesterol metabolism in cell lines infected with influenza A virus. Talanta 2010, 83, 262-268.

3. Xiao, J.F.; Zhou, B.; Ressom, H.W. Metabolite identification and quantitation in LC-MS/MS-based metabolomics. Trac-Trend Anal. Chem. 2012, 32, 1-14.

4. Lu, W.; Bennett, B.D.; Rabinowitz, J.D. Analytical strategies for LC-MS-based targeted metabolomics. J. Chromatogr. B 2008, 871, 236-242.

5. Ramautar, R.; Somsen, G.W.; de Jong, G.J. CE-MS for metabolomics: Developments and applications in the period 2010-2012. Electrophoresis 2013, 34, 86-98.

6. Celebier, M.; Ibanez, C.; Simo, C.; Cifuentes, A. A Foodomics approach: CE-MS for comparative metabolomics of colon cancer cells treated with dietary polyphenols. Methods Mol. Biol. 2012, $869,185-195$.

7. Ramautar, R.; Somsen, G.W.; de Jong, G.J. CE-MS in metabolomics. Electrophoresis 2009, 30, 276-291.

8. Weckwerth, W.; Morgenthal, K. Metabolomics: From pattern recognition to biological interpretation. Drug Discovery Today 2005, 10, 1551-1558.

9. Mashego, M.R.; Rumbold, K.; De Mey, M.; Vandamme, E.; Soetaert, W.; Heijnen, J.J. Microbial metabolomics: Past, present and future methodologies. Biotechnol. Lett. 2007, 29, 1-16.

10. Jagerdeo, E.; Montgornery, M.A.; Sibum, M.; Sasaki, T.A.; LeBeau, M.A. Rapid Analysis of Cocaine and Metabolites in Urine Using a Completely Automated Solid-Phase Extraction-High-Performance Liquid Chromatography-Tandem Mass Spectrometry Method. J. Anal. Toxicol. 2008, 32, 570-576.

11. Idborg, H.; Zamani, L.; Edlund, P.O.; Schuppe-Koistinen, I.; Jacobsson, S.P. Metabolic fingerprinting of rat urine by LC/MS Part 1. Analysis by hydrophilic interaction liquid chromatography-electrospray ionization mass spectrometry. J. Chromatogr. B 2005, 828, 9-13.

12. Stefan Blech, R.L. Resolving the microcosmos of complex samples: UPLC/travelling wave ion mobility separation high resolution mass spectrometry for the analysis of in vivo drug metabolism studies. Int. J. Ion. Mob. Spec. 2013, 16, 5-17.

13. Kind, T.; Fiehn, O. Advances in structure elucidation of small molecules using mass spectrometry. Bioanal. Rev. 2010, 2, 23-60.

14. Thomas, M.C.; Dunn, S.R.; Altvater, J.; Dove, S.G.; Nette, G.W. Rapid identification of long-chain polyunsaturated fatty acids in a marine extract by HPLC-MS using data-dependent acquisition. Anal. Chem. 2012, 84, 5976-5983.

15. Oberacher, H.; Schubert, B.; Libiseller, K.; Schweissgut, A. Detection and identification of drugs and toxicants in human body fluids by liquid chromatography-tandem mass spectrometry under data-dependent acquisition control and automated database search. Anal. Chim. Acta 2013, 770, $121-131$.

16. Thalassinos, K.; Vissers, J.P.; Tenzer, S.; Levin, Y.; Thompson, J.W.; Daniel, D.; Mann, D.; DeLong, M.R.; Moseley, M.A.; America, A.H.; et al. Design and application of a data-independent precursor and product ion repository. J. Am. Soc. Mass Spectrom. 2012, 23, 1808-1820.

17. Bateman, K.P.; Castro-Perez, J.; Wrona, M.; Shockcor, J.P.; Yu, K.; Oballa, R.; Nicoll-Griffith, D.A. MSE with mass defect filtering for in vitro and in vivo metabolite identification. Rapid Commun. Mass Spectrom. 2007, 21, 1485-1496. 
18. Castro-Perez, J.M.; Kamphorst, J.; DeGroot, J.; Lafeber, F.; Goshawk, J.; Yu, K.; Shockcor, J.P.; Vreeken, R.J.; Hankemeier, T. Comprehensive LC-MSE Lipidomic Analysis using a Shotgun Approach and Its Application to Biomarker Detection and Identification in Osteoarthritis Patients. J. Proteome Res. 2010, 9, 2377-2389.

19. Plumb, R.S.; Johnson, K.A.; Rainville, P.; Smith, B.W.; Wilson, I.D.; Castro-Perez, J.M.; Nicholson, J.K. UPLC/MS(E); A new approach for generating molecular fragment information for biomarker structure elucidation. Rapid Commun. Mass Spectrom. 2006, 20, 1989-1994.

20. Zhu, M.; Zhang, H.; Humphreys, W.G. Drug metabolite profiling and identification by high-resolution mass spectrometry. J. Biol. Chem. 2011, 286, 25419-25425.

21. Zhao, Y.Y.; Cheng, X.L.; Wei, F.; Bai, X.; Tan, X.J.; Lin, R.C.; Mei, Q. Intrarenal metabolomic investigation of chronic kidney disease and its TGF-betal mechanism in induced-adenine rats using UPLC Q-TOF/HSMS/MS(E). J. Proteome Res. 2013, 12, 692-703.

22. Zhao, Y.-Y.; Shen, X.; Cheng, X.-L.; Wei, F.; Bai, X.; Lin, R.-C. Urinary metabonomics study on the protective effects of ergosta-4,6,8(14),22-tetraen-3-one on chronic renal failure in rats using UPLC Q-TOF/MS and a novel MSE data collection technique. Process Biochem. 2012, 47, 1980-1987.

23. Pieroni, L.; Finamore, F.; Ronci, M.; Mattoscio, D.; Marzano, V.; Mortera, S.L.; Quattrucci, S.; Federici, G.; Romano, M.; Urbani, A. Proteomics investigation of human platelets in healthy donors and cystic fibrosis patients by shotgun nUPLC-MSE and 2DE: A comparative study. Mol. Biosyst. 2011, 7, 630-639.

24. Hummel, M.; Cordewener, J.H.; de Groot, J.C.; Smeekens, S.; America, A.H.; Hanson, J. Dynamic protein composition of Arabidopsis thaliana cytosolic ribosomes in response to sucrose feeding as revealed by label free MSE proteomics. Proteomics 2012, 12, 1024-1038.

25. Walles, M.; Gauvin, C.; Morin, P.E.; Panetta, R.; Ducharme, J. Comparison of sub-2- $\mu$ m particle columns for fast metabolite ID. J. Sep. Sci. 2007, 30, 1191-1199.

26. Castro-Perez, J.; Roddy, T.P.; Nibbering, N.M.M.; Shah, V.; McLaren, D.G.; Previs, S.; Attygalle, A.B.; Herath, K.; Chen, Z.; Wang, S.P.; et al. Localization of fatty acyl and double bond positions in phosphatidylcholines using a dual stage CID fragmentation coupled with ion mobility mass spectrometry. J. Am. Soc. Mass Spectrom. 2011, 22, 1552-1567.

27. Bohrer, B.C.; Clemmer, D.E. Biologically-Inspired peptide reagents for enhancing IMS-MS analysis of carbohydrates. J. Am. Soc. Mass Spectrom. 2011, 22, 1602-1609.

28. Dwivedi, P.; Schultz, A.J.; Hill, H.H. Metabolic profiling of human blood by high-resolution ion mobility mass spectrometry (IM-MS). Int. J. Mass Spectrom. 2010, 298, 78-90.

29. Giles, K.; Williams, J.P.; Campuzano, I. Enhancements in travelling wave ion mobility resolution. Rapid Commun. Mass Spectrom. 2011, 25, 1559-1566.

30. Stevens, J.F.; Page, J.E. Xanthohumol and related prenylflavonoids from hops and beer: To your good health! Phytochemistry 2004, 65, 1317-1330.

31. Jirasko, R.; Holcapek, M.; Vrublova, E.; Ulrichova, J.; Simanek, V. Identification of new phase II metabolites of xanthohumol in rat in vivo biotransformation of hop extracts using high-performance liquid chromatography electrospray ionization tandem mass spectrometry. J. Chromatogr. A 2010, $1217,4100-4108$. 
32. Legette, L.L.; Moreno Luna, A.Y.; Reed, R.L.; Miranda, C.L.; Bobe, G.; Proteau, R.R.; Stevens, J.F. Xanthohumol lowers body weight and fasting plasma glucose in obese male Zucker fa/fa rats. Phytochemistry 2012, 91, 236-241.

33. Legette, L.; Ma, L.; Reed, R.L.; Miranda, C.L.; Christensen, J.M.; Rodriguez-Proteau, R.; Stevens, J.F. Pharmacokinetics of xanthohumol and metabolites in rats after oral and intravenous administration. Mol. Nutr. Food Res. 2012, 56, 466-474.

34. Kooijman, E.E.; Burger, K.N.J. Biophysics and function of phosphatidic acid: A molecular perspective. BBA-Mol. Cell Biol. L. 2009, 1791, 881-888.

35. Rancoule, C.; Attané, C.; Grès, S.; Fournel, A.; Dusaulcy, R.; Bertrand, C.; Vinel, C.; Tréguer, K.; Prentki, M.; Valet, M.; et al. Lysophosphatidic acid impairs glucose homeostasis and inhibits insulin secretion in high-fat diet obese mice. Diabetologia 2013, 56, 1394-1402.

36. Lee, Y.M.; Hsieh, K.H.; Lu, W.J.; Chou, H.C.; Chou, D.S.; Lien, L.M.; Sheu, J.R.; Lin, K.H. Xanthohumol, a Prenylated Flavonoid from Hops (Humulus lupulus), Prevents Platelet Activation in Human Platelets. Evid.-based Complement Altern. Med. 2012, Article ID 852362.

37. Wang, C.; Kong, H.; Guan, Y.; Yang, J.; Gu, J.; Yang, S.; Xu, G. Plasma phospholipid metabolic profiling and biomarkers of type 2 diabetes mellitus based on high-performance liquid chromatography/electrospray mass spectrometry and multivariate statistical analysis. Anal. Chem. 2005, 77, 4108-4116.

38. Fuchs, B.; Schiller, E.; Wagner, U.; Hantzschel, H.; Arnold, K. The phosphatidylcholine/lysophosphatidylcholine ratio in human plasma is an indicator of the severity of rheumatoid arthritis: Investigations by P-31 NMR and MALDI-TOF MS. Clin. Biochem. 2005, 38, 925-933.

39. Nozawa, H. Xanthohumol, the chalcone from beer hops (Humulus lupulus L.), is the ligand for farnesoid X receptor and ameliorates lipid and glucose metabolism in KK-A(y) mice. Biochem. Biophys. Res. Commun. 2005, 336, 754-761.

40. Mortensen, P.B. Formation and degradation of dicarboxylic acids in relation to alterations in fatty acid oxidation in rats. Biochim. Biophys. Acta 1992, 1124, 71-79.

41. Kirkwood, J.S.; Legette, L.L.; Miranda, C.L.; Jiang, Y.; Stevens, J.F. A metabolomics driven elucidation of the anti-obesity mechanisms of xanthohumol. J. Biol. Chem. 2013, doi:10.1074/jbc.M112.445452.

42. Dwivedi, P.; Wu, P.; Klopsch, S.J.; Puzon, G.J.; Xun, L.; Hill, H.H., Jr. Metabolic profiling by ion mobility mass spectrometry (IMMS). Metabolomics 2008, 4, 63-80.

43. Shimizu, A.; Ohe, T.; Chiba, M. A novel method for the determination of the site of glucuronidation by ion mobility spectrometry-mass spectrometry. Drug Metab. Dispos. 2012, 40, 1456-1459.

44. Williams, M.D.; Reeves, R.; Resar, L.S.; Hill, H.H., Jr. Metabolomics of colorectal cancer: Past and current analytical platforms. Anal. Bioanal. Chem.2013, 405, 5013-5030.

45. Ridenour, W.B.; Kliman, M.; McLean, J.A.; Caprioli, R.M. Structural characterization of phospholipids and peptides directly from tissue sections by MALDI traveling-wave ion mobility-mass spectrometry. Anal. Chem. 2010, 82, 1881-1889.

46. Kliman, M.; May, J.C.; McLean, J.A. Lipid analysis and lipidomics by structurally selective ion mobility-mass spectrometry. BBA-Mol. Cell Biol. L. 2011, 1811, 935-945. 
47. Li, H.L.; Giles, K.; Bendiak, B.; Kaplan, K.; Siems, W.F.; Hill, H.H. Resolving structural isomers of monosaccharide methyl glycosides using drift tube and traveling wave ion mobility mass spectrometry. Anal. Chem. 2012, 84, 3231-3239.

48. Clowers, B.H.; Dwivedi, P.; Steiner, W.E.; Hill, H.H.; Bendiak, B. Separation of sodiated isobaric disaccharides and trisaccharides using electrospray ionization-atmospheric pressure ion mobility-time of flight mass spectrometry. J. Am. Soc. Mass Spectr. 2005, 16, 660-669.

49. Kaplan, K.A.; Chiu, V.M.; Lukus, P.A.; Zhang, X.; Siems, W.F.; Schenk, J.O.; Hill, H.H. Neuronal metabolomics by ion mobility mass spectrometry: Cocaine effects on glucose and selected biogenic amine metabolites in the frontal cortex, striatum, and thalamus of the rat. Anal. Bioanal. Chem. 2013, 405, 1959-1968.

50. Tautenhahn, R.; Bottcher, C.; Neumann, S. Highly sensitive feature detection for high resolution LC/MS. BMC Bioinf. 2008, 9, 504.

51. Xia, J.G.; Mandal, R.; Sinelnikov, I.V.; Broadhurst, D.; Wishart, D.S. MetaboAnalyst 2.0-A comprehensive server for metabolomic data analysis. Nucleic Acids Res. 2012, 40, W127-W133.

52. Xia, J.G.; Psychogios, N.; Young, N.; Wishart, D.S. MetaboAnalyst: A web server for metabolomic data analysis and interpretation. Nucleic Acids Res. 2009, 37, W652-W660.

53. Zhou, B.; Wang, J.L.; Ressom, H.W. MetaboSearch: Tool for mass-based metabolite identification using multiple databases. Plos One 2012, 7, e40096.

(C) 2013 by the authors; licensee MDPI, Basel, Switzerland. This article is an open access article distributed under the terms and conditions of the Creative Commons Attribution license (http://creativecommons.org/licenses/by/3.0/). 